\title{
Potencialização do efeito metemoglobinizante da dapsona em ratos pela $\mathrm{N}$-acetilcisteína
}

\author{
Natália Valadares de Moraes, Mauricio Homem de Mello, Ana Maria de Souza, Suely Vilela \\ Sampaio, Regina Helena Costa Queiroz*
}

Departamento de Análises Clínicas, Toxicológicas e Bromatológicas, Faculdade de Ciências Farmacêuticas de Ribeirão Preto, Universidade de São Paulo, Ribeirão Preto.

*Correspondência:

R. H. C. Queiroz

Faculdade de Ciências Farmacêuticas de Ribeirão Preto

Departamento de Análises Clínicas,

Toxicológicas e Bromatológicas

Av. do Café, s/n

14040-903 - Ribeirão Preto - SP, Brasil

E-mail: rqueiroz@fcfrp.usp.br
Dapsona (DDS) (4,4'diaminodifenilsulfona), fármaco de escolha para o tratamento da hanseniase, freqüentemente induz anemia hemolítica e metemoglobinemia. A N-hidroxilação, uma de suas principais vias de biotransformação, é constantemente relacionada com a metemoglobinemia observada com o uso do fármaco. Com o objetivo de prevenir a hemotoxicidade induzida pela DDS, Nacetilcisteina, fármaco precursor de glutationa, foi administrada em associação com DDS em ratos machos Wistar pesando 220$240 \mathrm{~g}$. Os animais foram anestesiados e o sangue coletado da aorta para determinação da concentração plasmática de DDS por CLAE, determinação dos nivveis de metemoglobina e de glutationa eritrocitária por espectrofotometria, e avaliação de parâmetros bioquímicos e hematológicos. Os resultados obtidos mostraram que a $N$-acetilcisteina potenciou o efeito metemoglobinizante da dapsona devido ao aumento de sua concentração plasmática e conseqüente aumento da formação da N-hidroxilamina. Concluímos que as interações medicamentosas com a dapsona exigem estudos individualizados a fim de evitar os efeitos adversos do fármaco.

\section{INTRODUÇÃO}

A hanseníase é uma doença infecciosa crônica causada pelo patógeno Mycobacterium leprae. Até 1980, a dapsona (4,4'-diaminodifenilsulfona) era o fármaco de escolha para o tratamento da hanseníase. Em 1981, o Comitê de Peritos em Quimioterapia da Organização Mundial da Saúde (OMS) definiu novos esquemas terapêuticos, devido à dificuldade no controle de bacilos resistentes com o emprego de medicamentos administrados isoladamente
(Cambau et al., 1997). A associação de dapsona (DDS), rifampicina e clofazimina no tratamento da hanseníase permitiu um controle mais efetivo na transmissão e evolução da doença. Apesar dos esforços da OMS, aproximadamente $94 \%$ dos casos conhecidos e dos novos casos diagnosticados nas Américas são notificados no Brasil. Ao longo dos últimos anos, as taxas de prevalência têm declinado consideravelmente com redução de até $20 \%$ ao ano, resultado da consolidação do tratamento politerápico. Entretanto, as taxas de detecção de casos novos ainda são eleva- 
das, e a expectativa é que só se alcance a meta de eliminação da doença quando a prevalência for inferior a 1/10.000 habitantes (Cambau et al., 1997; Brasil MS, 1998, 1999; Araujo, 2003; OPAS, 2004).

Os efeitos adversos relacionados aos esquemas terapêuticos empregados na hanseníase mais freqüentes são a metemoglobinemia e a anemia hemolítica associados à exposição a DDS (Coleman, 1995; Landers et al., 1996; Queiroz et al., 1997; Ward, McCarty, 1998; Salamat, Watson, 2003). Outros efeitos adversos também citados, porém com menor freqüência, são: letargia, granulocitopenia, problemas hepáticos e renais, dermatite esfoliativa, neuropatia periférica, febre, cefaléias, psicoses e fotodermatite (Pavithran, Satish, 1997; Leslie et al., 2003).

A dapsona possui também atividade contra malária e contra pneumonia causada pelo Pneumocystis carinii. Outro uso da dapsona se dá em situações inflamatórias como na dermatite herpetiforme (Coleman, Jacobus, 1993). O uso deste medicamento, porém, é freqüentemente limitado, devido aos seus efeitos adversos.

No homem, a DDS é extensivamente biotransformada, e sua N-hidroxilação é a responsável pelos efeitos adversos no sistema hematológico, tais como a metemoglobinemia e a anemia hemolítica (Tingle et al., 1990; Grossman et al., 1992). A metemoglobinemia descreve o estado clínico em que mais de $1 \%$ da hemoglobina do sangue foi oxidada à forma férrica, diminuindo a capacidade de transporte de oxigênio. Clinicamente, a toxicidade relacionada a metemoglobina é ligada aos seus teores no sangue. Dispnéia, náuseas e taquicardia ocorrem quando há níveis de até $30 \%$; letargia, estupor e perda de consciência resultam de níveis de aproximadamente $50 \%$; níveis de 50 a 70\% levam a arritmias cardíacas, falência circulatória e depressão neurológica, e níveis acima de 70\% geralmente levam à morte (Coleman, Coleman, 1996).

Várias tentativas estão sendo testadas em virtude de aumentar a tolerância dos pacientes à dapsona. Entre elas, foram incluídas a administração de vitaminas $\mathrm{E}$ e C com dapsona (Prussick et al., 1992). No entanto, esses antioxidantes causaram pequeno impacto na metemoglobinemia induzida pelo fármaco. Desta forma, o presente trabalho tem como objetivo avaliar a eficácia da Nacetilcisteína na prevenção da formação de metemoglobina induzida pela dapsona em ratos. AN-acetilcisteína (NAC), fármaco mucolítico, é um precursor da glutationa $(\mathrm{GSH})$ utilizado no tratamento da bronquite crônica e outras patologias pulmonares. Trata-se de um composto tiólico que atinge o meio intracelular e é fonte potencial de equivalentes redutores (Lehninger et al., 1995). A GSH auxilia na manutenção de grupos sulfidrilas de proteínas no seu estado reduzido e o ferro do grupo heme no estado ferroso $\left(\mathrm{Fe}^{2+}\right)$. O re- sultado esperado é que a interação com a $\mathrm{N}$-acetilcisteína agregue ao efeito farmacológico da dapsona, um menor risco de reações adversas principalmente a metemoglobinemia e anemia hemolítica, reações dose-dependentes.

\section{MATERIAL E MÉTODOS}

Quatro grupos de ratos Wistar ( $\mathrm{n}=8$ ), machos, adultos jovens (220-240 g), mantidos em gaiolas plásticas ( 50 × 35 x $15 \mathrm{~cm}$ ), em ciclo controlado de 12 horas de claro e escuro, com água e comida fornecidos "ad libitum” receberam intraperitonealmente os fármacos solubilizados em dimetilsulfóxido (DMSO), durante 5 dias. Os grupos controle, NAC, DDS e DDS + NAC receberam, respectivamente: $200 \mu \mathrm{L}$ de DMSO; $200 \mu \mathrm{L}$ de N-acetilcisteína $75 \mathrm{mg} / \mathrm{kg}$ (Ajinomoto) em DMSO; $200 \mu \mathrm{L}$ de dapsona $40 \mathrm{mg} / \mathrm{kg}$ (Sigma) em DMSO; e $200 \mu \mathrm{L}$ de N-acetilcisteína $75 \mathrm{mg} / \mathrm{kg}$ e dapsona $40 \mathrm{mg} / \mathrm{kg}$ nos tempos 0, 24, 48, 72 e $96 \mathrm{~h}$.

No quinto dia de tratamento, duas horas após a administração dos fármacos, os animais foram anestesiados com isofluorano, e o sangue foi colhido da artéria aorta e fracionado em três tubos: um contendo 100 UI de heparina (determinação de metemoglobina, glutationa e concentração plasmática de dapsona); outro contendo EDTA $\cdot \mathrm{K}_{2} 10 \%$ na proporção de $1 \mathrm{mg} / \mathrm{mL}$ de sangue (parâmetros hematológicos); e outro sem anticoagulante para obtenção de soro (parâmetros bioquímicos). O protocolo experimental foi aprovado pela Comissão de Ética no Uso de Animais (CEUA) do Campus de Ribeirão Preto.

\section{Determinação da concentração plasmática de dapsona por cromatografia líquida de alta eficiência}

A determinação plasmática da dapsona foi realizada através de cromatografia líquida de alta eficiência (CLAE) segundo o método descrito por Queiroz et al. (1997b). Foi utilizado um cromatógrafo líquido Shimadzu - modelo LC$10 \mathrm{AS}$, equipado com injetor Rheodyne modelo 7125 , amostrador de $100 \mu \mathrm{L}$, coluna de fase reversa $(\mathrm{C} 8$, LiChrocart ${ }^{\circledR} 100$ - Merck - 125 x $4 \mathrm{~mm}$, partículas de $5 \mu \mathrm{m}$ ), detector por absorbância no ultravioleta (Shimadzu modelo SPD-10A) operando em $286 \mathrm{~nm}$. A fase móvel foi constituída de água:metanol ( $65: 35 \mathrm{v} / \mathrm{v})$, o fluxo utilizado foi de $1,2 \mathrm{~mL} / \mathrm{min}$. Os cromatogramas foram obtidos em integrador Shimadzu - C - R6A chromatopac.

\section{Determinação de metemoglobina}

A determinação dos níveis de metemoglobina foi realizada nas amostras de sangue heparinizado, pelo método de Evelyn \& Malloy modificado conforme descrito por 
Harisson \& Jollow (1986) utilizando o espectrofotômetro.

\section{Determinação da glutationa eritrocitária}

As amostras de sangue heparinizado foram analisadas conforme o método descrito por Beutler et al. (1963) para a determinação espectrofotométrica de glutationa eritrocitária, no espectro do ultravioleta (412 nm).

\section{Parâmetros hematológicos}

A contagem de eritrócitos foi feita por microscopia ótica em câmara de Neubauer, usando a solução de Hayem como diluente. $\mathrm{O}$ hematócrito foi determinado usando uma centrífuga para microhematócrito Fanem e tubos capilares com diâmetro uniforme. A concentração de hemoglobina foi determinada pelo método colorimétrico da cianometemoglobina. Os índices eritrocitométricos (volume corpuscular médio - VCM, hemoglobina corpuscular média - HCM e concentração de hemoglobina corpuscular média - CHCM) foram calculados a partir dos valores determinados para número de eritrócitos, concentração de hemoglobina e hematócrito. O número de reticulócitos foi determinado em extensão sanguínea corada com azul cresil brilhante (Fimls, 1989). Os níveis séricos de haptoglobina foram determinados por nefelometria utilizando kit e nefelômetro Dade Behring.

\section{Parâmetros bioquímicos}

Os níveis séricos dos parâmetros bioquímicos foram quantificados por técnicas espectrofotométricas e reagentes da marca Labtest Diagnóstica, sendo: atividade da enzima lactato desidrogenase (LDH) pelo método cinético com medida de ponto-final, e bilirrubina total e direta pelo método colorimétrico, segundo Sims \& Horn (1958). A bilirrubina indireta foi calculada através da diferença entre bilirrubina total e direta.

\section{Análise estatística}

Os testes foram realizados com auxílio do software GraphPad Instat ${ }^{\circledR}$, para o estudo da análise das médias (ANOVA) e análise de comparação múltipla entre os grupo (Tukey-Kramer). O nível de significância foi fixado em $\mathrm{p}<0,05$.

\section{RESULTADOS E DISCUSSÃO}

Os estudos iniciaram-se com o grupo controle que apresentou valores de metemoglobina no sangue na ordem de 0,31 a $0,73 \%$. Assim fixamos o valor de referência da porcentagem de metemoglobina para ratos em $0,49 \pm$ 0,18\%. Outros veículos, como o cremophor, acarretaram desidratação no animal por diarréia, e, portanto, foi mantido o DMSO como veículo.

Em estudos realizados em dose única no laboratório, a dose de $40 \mathrm{mg} / \mathrm{kg}$ de DDS foi a que apresentou efeito metemoglobinizante mais evidente. Já a dose de NAC que melhor apresentou resposta, sem induzir metemoglobinemia significativa, foi a de $75 \mathrm{mg} / \mathrm{kg}$. No grupo DDS em dose múltipla de $40 \mathrm{mg} / \mathrm{kg}$, o valor médio de metemoglobina foi de $12,45 \pm 1,77 \%$. O grupo NAC, em dose múltipla de 75 $\mathrm{mg} / \mathrm{kg}$ apresentou $1,88 \pm 0,15 \%$ de metemoglobina.

Surpreendentemente, ao iniciar a administração da associação de NAC (75 mg/kg) e DDS (40 mg/kg), os níveis de metemoglobina, ao invés de diminuírem, aumentaram de $12,45 \pm 1,77 \%$ (DDS) para 18,63 $\pm 1,53 \%$ (DDS + NAC) (Figura 1). Verificou-se então que a associação, ao invés de proteger, potenciou a oxidação do ferro da hemoglobina.

Kramer et al. em 1972, trabalhando com anilinas em experimentos semelhantes, propuseram que no processo de formação da metemoglobina, os derivados N-hidroxilados são convertidos a moléculas instáveis, os nitrosoarenos. Estes, por sua vez, podem regenerar as N-hidroxilaminas através do poder redutor da enzima NADPH-metemoglobina redutase ou da glutationa eritrocitária, possibilitando a oxidação de outras moléculas de hemoglobina e a formação de um ciclo redox. Cada molécula de hidroxilamina é capaz de oxidar até cinco moléculas de oxiemoglobina, e o ciclo somente cessa quando a glutationa eritrocitária está quase completamente depletada. Kramer et al. (1972) sugerem ainda que o processo de regeneração da Nhidroxilamina a partir do nitrosoareno não requer catálise enzimática. Para isso, basearam-se no fato de a formação de metemoglobina ocorrer de forma significativa quando são adicionados compostos redutores como GSH ou NADPH à hemoglobina, onde não há atividade da enzima NADPH-metemoglobina redutase.

Tal mecanismo também foi sugerido por Coleman \& Jacobus (1993) em estudo in vitro. Estes autores sugerem que o composto nitrosoareno, formado após a ação oxidativa da hidroxilamina sobre a hemoglobina, pode sofrer ação da glutationa de duas formas distintas: pode reduzir o nitrosoareno até o precursor hidroxilado ou pode reagir com este composto formando um derivado sulfenamida. No primeiro caso, quando há a formação da hidroxilamina a partir do nitrosoareno, o processo de formação de metemoglobina se reinicia, formando assim um ciclo redox que só termina quando os níveis de glutationa são reduzidos. Dentro das células, forma-se um delicado equilíbrio entre a velocidade de regeneração da dapsona hidroxilamina e da for- 


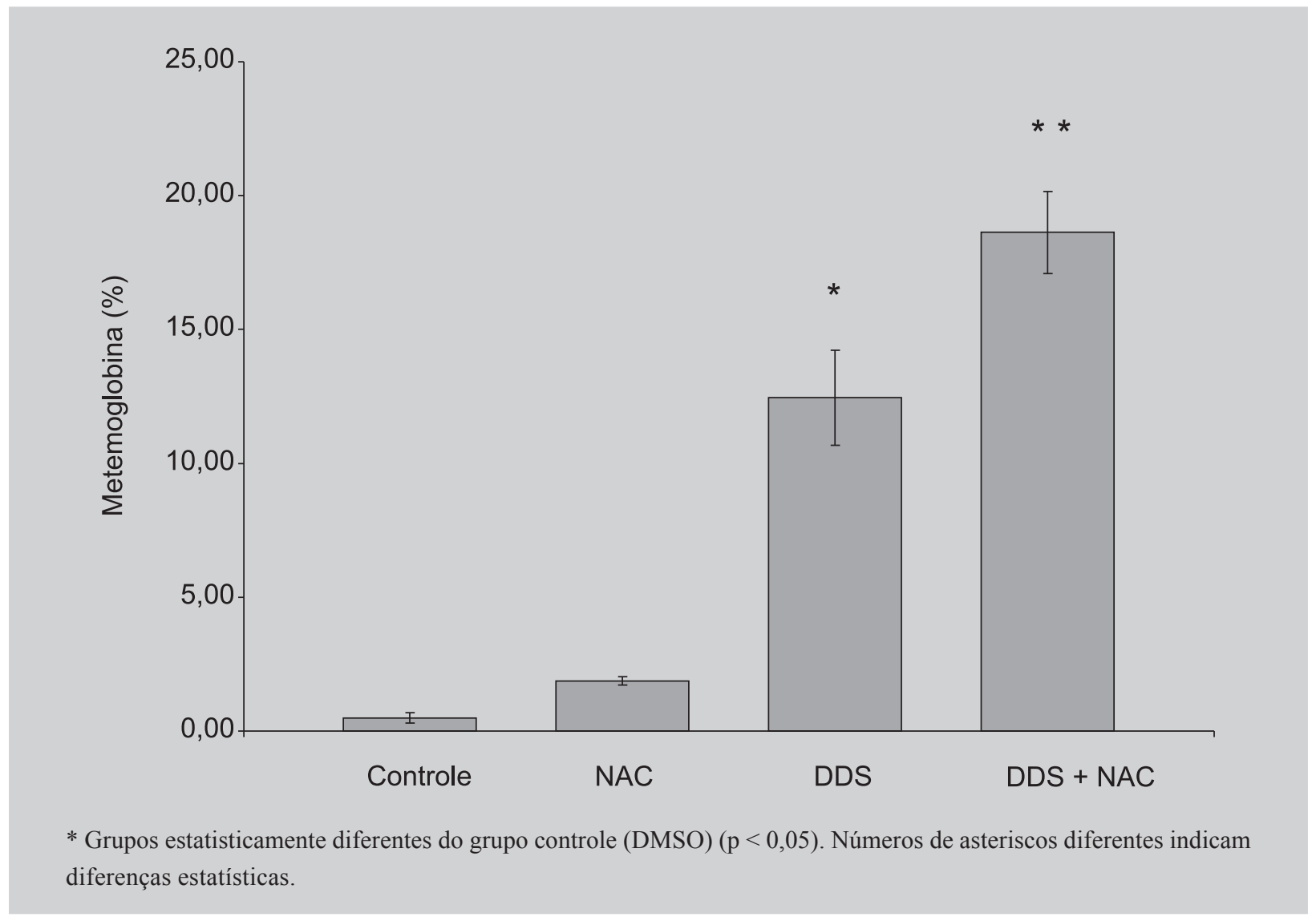

FIGURA 1 - Porcentagens de metemoglobina dos grupos de animais tratados com dimetilsulfóxido (DMSO; grupo controle); N-acetilcisteína $75 \mathrm{mg} / \mathrm{kg}$ (NAC); dapsona $40 \mathrm{mg} / \mathrm{kg}$ (DDS); associação dapsona $40 \mathrm{mg} / \mathrm{kg}$ e N-acetilcisteína $75 \mathrm{mg} / \mathrm{kg}$ (DDS + NAC)

mação de metemoglobina. Os fatores que afetam este equilíbrio podem alterar a formação de metemoglobina induzida pela DDS. A N-acetilcisteína, por ser precursora da glutationa, altera tal equilíbrio proporcionando maior capacidade de regeneração da N-hidroxilamina (Figura 2). Sendo assim, a formação de metemoglobina é consideravelmente maior nos animais tratados com $\mathrm{N}$-acetilcisteína em associação com a dapsona quando comparamos aos animais tratados somente com a dapsona. Por outro lado, quando o derivado nitrosoareno eventualmente não participa desse ciclo redox, pode se ligar a glutationa ou outros grupos tióis formando um derivado sulfenamida ou outros adutos de estabilidade variável. A hidrólise desse adutos ou da sulfenamida libera então a amina livre (dapsona), que se difunde para fora do eritrócito.

Por esta razão, a concentração plasmática de dapsona aumenta de 189,64 $\pm 14,42 \mu \mathrm{g} / \mathrm{mL}$ (grupo DDS) para $329,14 \pm 56,30 \mu \mathrm{g} / \mathrm{mL}$ (grupo DDS + NAC), e o fármaco pode novamente ser metabolisado até os compostos N-hidroxilados.
Em relação à determinação de glutationa, não observamos diferença estatística quando comparamos o grupo controle $(65,04 \pm 2,66)$ com o grupo de animais tratados com $\mathrm{N}$-acetilcisteína $(70,00 \pm 5,53)$. Apenas os grupos DDS e DDS + NAC, apresentaram níveis de glutationa eritrocitária significativamente maiores quando comparados ao grupo controle (controle: $65,04 \pm 2,66$; DDS: 90,90 $\pm 6,08$; DDS + NAC: $97,06 \pm 4,46$ ) (Figura 3). Portanto, os níveis de glutationa não auxiliam na proteção do efeito metemoglobinizante da dapsona. Mesmo com o uso de um fármaco oxidante, como a dapsona, não é possível detectar a diminuição da glutationa eritrocitária no grupo DDS, visto que, a diminuição de glutationa é acompanhada pela formação de altos níveis de glutationa oxidada (GSSG) e esta pode ser rapidamente reduzida pela GSSG redutase (Meister, 1991). Nesse processo os níveis de GSH são recuperados em menos de 10 minutos (Costagliola, 1990), sendo que $98 \%$ do peptídeo encontra-se na forma reduzida (Meister, Anderson, 1983). Essa recuperação é rápida, e muitas tentativas de depletar o estoque de GSH promovem, 


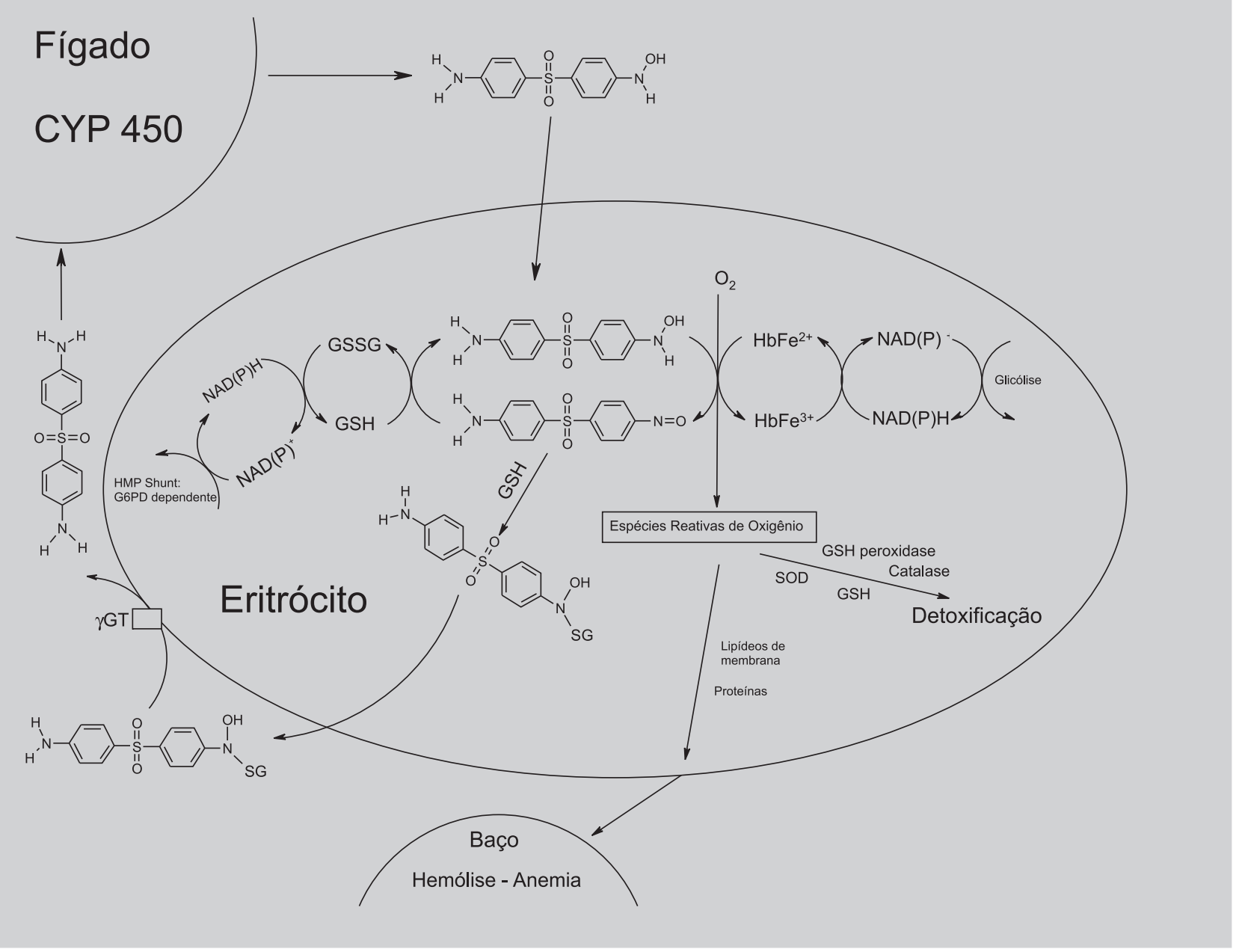

FIGURA 2 - Interação medicamentosa entre N-acetilcisteína e dapsona

paradoxalmente, um aumento nos níveis de GSH (Meister, 1991).

Apesar de alguns parâmetros hematológicos e bioquímicos apresentarem diferenças estatisticamente significantes entre os grupos, estas variações parecem não ter significância biológica, não podendo ser caracterizadas como efeitos adversos (Tabela 1). O aumento significante da bilirrubina e discreta diminuição da haptoglobina em relação ao grupo controle indicam maior destruição de eritrócitos, especialmente no grupo DDS + NAC, que também foi o que demonstrou metemoglobinemia mais intensa. Considerando que as anemias hemolíticas são normocrômicas normocíticas (Fimls, 1989), os índices eritrocitométricos devem permanecer dentro da faixa de normalidade, conforme o observado. Seria esperado um aumento do número de reticulócitos, o que não foi observado, pois o grau de hemólise nos grupos estudados pareceu pequeno.

\section{CONCLUSÕES}

Pelos resultados obtidos, pode-se concluir que a administração de $\mathrm{N}$-acetilcisteína promoveu uma interação farmacocinética com a dapsona, alterando de forma significativa sua concentração plasmática. O aumento da concentração plasmática de dapsona resultante dessa interação, por sua vez, potenciou o efeito metemoglobinizante da dapsona.

A interação entre fármacos representa um papel importante na terapia farmacológica, sejam essas interações de origens farmacodinâmicas ou farmacocinéticas, representando ora forma de tratamento, como na associação do paracetamol com a $\mathrm{N}$-acetilcisteína, ora fonte de reações adversas, com conseqüências clínicas significativas.

Neste contexto deve ser reforçada a importância de mais estudos com o propósito de trazer maior segurança à utilização da dapsona, pois esta pode ter seu uso clíni- 


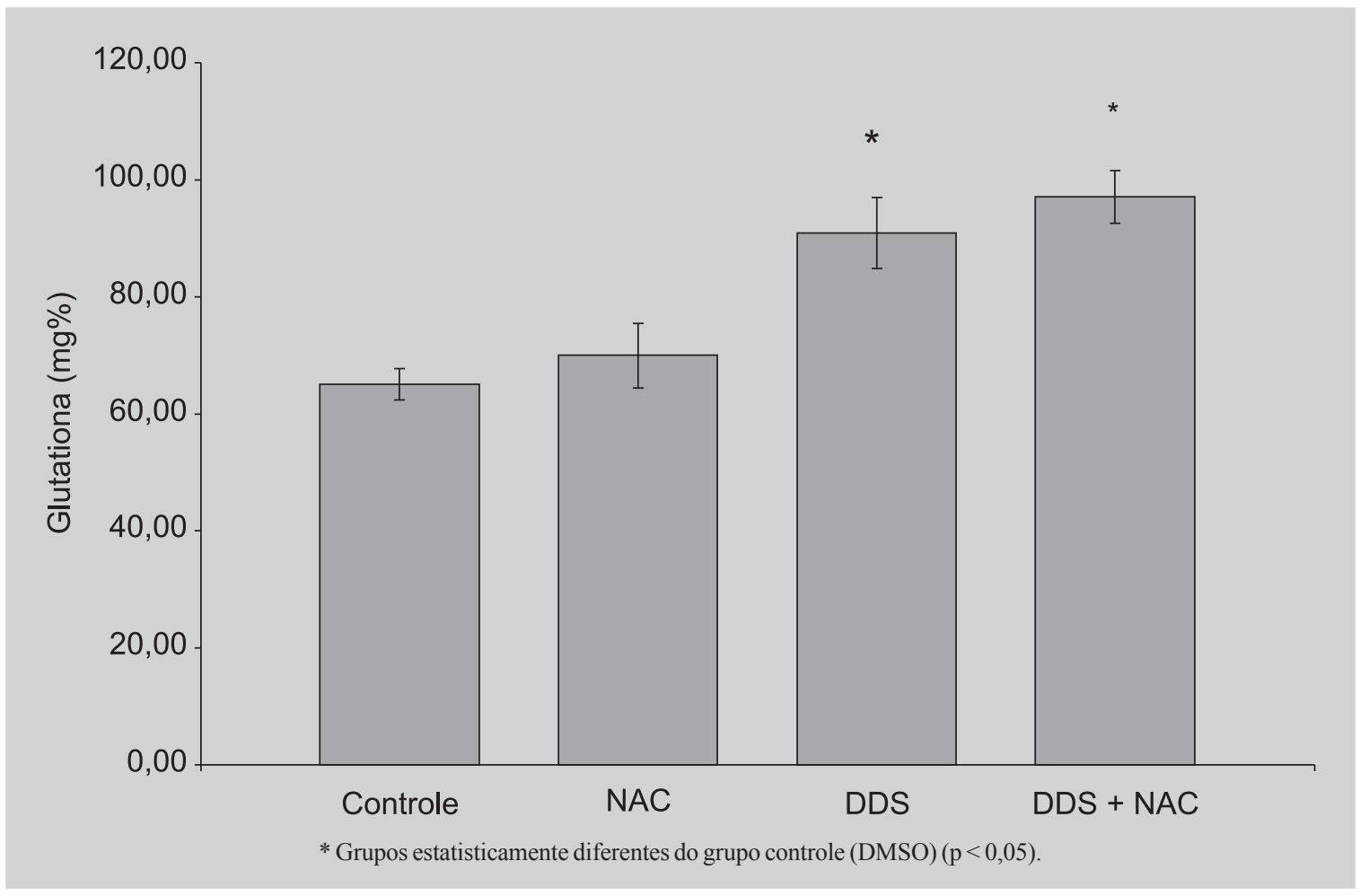

FIGURA 3 - Níveis de glutationa eritrocitária ( $\mathrm{mg} \%)$ no sangue dos grupos de animais tratados com dimetilsulfóxido (DMSO; grupo controle); N-acetilcisteína $75 \mathrm{mg} / \mathrm{kg}$ (NAC); dapsona $40 \mathrm{mg} / \mathrm{kg}$ (DDS); associação dapsona $40 \mathrm{mg} / \mathrm{kg}$ e N-acetilcisteína $75 \mathrm{mg} / \mathrm{kg}$ (DDS + NAC).

TABELA 1 - Valores dos parâmetros bioquímicos e hematológicos em animais que receberam N-acetilcisteína (NAC) $75 \mathrm{mg} / \mathrm{kg}$ e dapsona (DDS) $40 \mathrm{mg} / \mathrm{kg}$

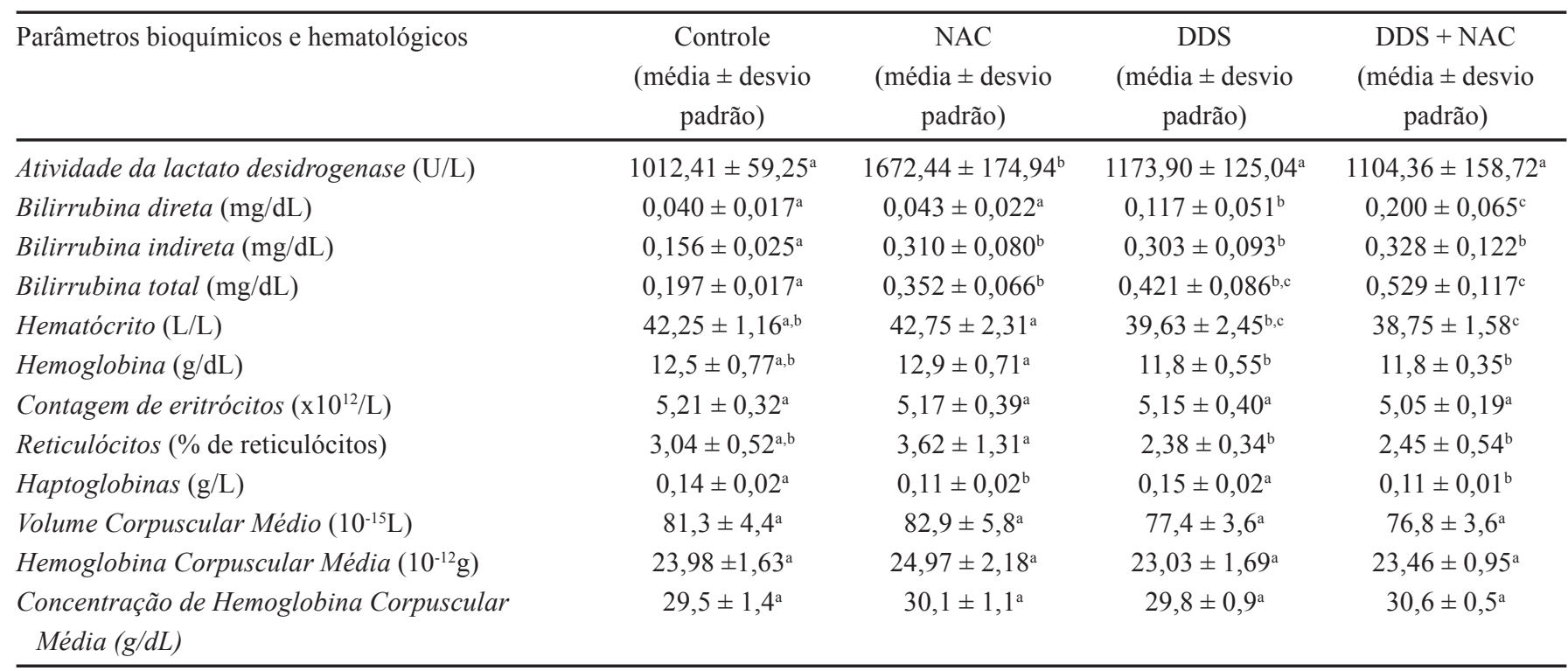

Resultados expressos como média \pm desvio padrão $(\mathrm{n}=8)$. Diferentes letras sobrescritas indicam diferenças estatísticas $(\mathrm{p}<0,05)$. Controle: grupo tratado com o veículo dimetilsulfóxido (DMSO); NAC: grupo tratado com $75 \mathrm{mg} / \mathrm{kg}$ de N-acetilcisteína; DDS: animais tratados com $40 \mathrm{mg} / \mathrm{kg}$ de dapsona; DDS + NAC: animais tratados com $40 \mathrm{mg} / \mathrm{kg}$ de dapsona e $75 \mathrm{mg} / \mathrm{kg}$ de $\mathrm{N}$-acetilcisteína. Os fármacos foram administrados intraperitonealmente. 
co ampliado, além de promover uma melhora na qualidade de vida de pacientes hansenianos em tratamento de longo prazo.

\section{AGRADECIMENTOS}

Os autores agradecem o suporte técnico de Sônia Aparecida Carvalho Dreossi, Maria Aparecida Buzeto Pereira, Zita Maria de Oliveira Gregório e Adélia Cristina de Oliveira Cintra. Os autores agradecem ainda ao Prof. Dr. Osvaldo de Freitas por fornecer N-acetilcisteína.

Agradecemos à Fundação de Amparo à Pesquisa do Estado de São Paulo (FAPESP 2003/11987-9), pelo apoio financeiro.

\section{ABSTRACT}

\section{Potentiation of dapsone induced methemoglobinemia by $\mathrm{N}$-acetylcysteine in rats}

Dapsone (DDS) (4,4'diaminodiphenylsulfone), the drug of choice for the treatment of leprosy, frequently induces hemolytic anemia and methemoglobinemia. $N$-hydroxylation, one of the major pathways of biotransformation, has been constantly related to the methemoglobinemia after the use of the drug. In order to prevent the dapsone-induced hemotoxicity, $N$-acetylcysteine, a drug precursor of glutathione, was administered in combination with DDS to male Wistar rats, weighting 220-240 g. The animals were then anaesthetized and blood was collected from the aorta for determination of plasma DDS concentration by HPLC, determination of methemoglobinemia and glutathione by spectrophotometry, and for biochemical and hematological parameters. Our results showed that $N$-acetylcysteine enhanced dapsone-induced methemoglobinemia due to increased dapsone plasmatic concentration and consequent increased $N$-hydroxylamine formation. We concluded that drug interactions with dapsone require individually studies in order to avoid undesirable effects of dapsone

UNITERMS: Dapsone. N-acetylcysteine. Glutathione. Methemoglobinemia.

\section{REFERÊNCIAS BIBLIOGRÁFICAS}

ARAUJO, M.G. Leprosy in Brasil. Rev. Soc. Bras. Med. Trop., Uberaba, v.36, n.3, p.373-382, 2003.

BEUTLER, E.; DURON, O.; KELLY, B. M. Improved method for the determination of blood glutathione. J. Lab. Clin. Med., St. Louis, v.61, n.5, p.882-888, 1963.
BRASIL. Ministério da Saúde. Secretaria de Políticas de Saúde. DGPE. Hanseníase no Brasil: Progressos e dificuldades em relação à eliminação. Brasília: DGPE, 1998. 5p.

BRASIL. Ministério da Saúde. Secretaria de Políticas de Saúde. DGPE. Guia para implantar/implementar as atividades de controle de hanseníase nos planos estaduais e municipais de saúde. Brasília: DGPE, 1999. 28p.

CAMBAU, E.; PERANI, E.; GUILLEMIN, I.; JAMET, P.; JI, B. Multidrug-resistance to dapsone, rifampicin, and ofloxacin in Mycobacterium leprae. Lancet, Londres, v.349, p.103-104, 1997.

COLEMAN, M. D.; JACOBUS, D. P. Reduction of dapsone hydroxylamine to dapsone during methemoglobin formation in human erythrocytes in vitro. Biochem. Pharmacol., Oxford, v.45, n.5, p.1027-1033, 1993.

COLEMAN, M.D. Dapsone toxicity: some current perspectives. Gen. Pharmacol., Oxford, v.26, n.7, p.1461-1467, 1995.

COLEMAN, M. D.; COLEMAN, N.A. Drug-induced methaemoglobinaemia. Drug Saf., Auckland, v.14, p.394405, 1996.

COSTAGLIOLA, C. Oxidative state of glutathione in red blood cells and plasma of diabetic patients: in vivo and in vitro study. Clin. Physiol. Biochem., Basel, v.8, p.204210, 1990.

FIMLS, A. S. Hematology: a combined theoretical \& technical approach. Philadelphia: WB Saunders Co. 1989. p. 203-228.

GROSSMAN, S. J.; SIMSON, J.; JOLLOW, D. J. Dapsoneinduced hemolytic anemia: effect of N-hydroxy dapsone on the sulfhydryl status and membrane proteins of rat erythrocytes. Toxicol. Appl. Pharmacol., Nova York, v.117, p.208-217, 1992.

HARRISON, H. J.; JOLLOW, J. D. Role of aniline metabolites in aniline-induced hemolytic anemia. $J$. Pharmacol. Exp. Ther., Baltimore, v.238, n.3, p.1045-54, 1992.

HENRY, R. J.; CANNON, D. C.; WINKELMAN, J. W. Quimica Clinica: bases y técnicas. 2. ed. Barcelona: Editorial JJMS, 1980. v.1-2. 
KAPLAN, L. A.; PESCE, A. J. Clinical Chemistry: Theory, Analysis and Correlation. 3 ed. St. Louis: C. v. Mosby, 1996. 1064p.

KRAMER, P. A.; GLADER, B. E.; LI, T. K. Mechanism of methemoglobin formation by diphenylsulfones. Biochem. Pharmacol., Oxford, v.21, p.1265-1274, 1972.

LANDERS, D.; BERGIN, C.; O'LEARY, A.; MERRY, C.; KEATING, S.; MULCAHY, F. Dapsone induced methaemoglobinaemia. Int. J. STD AIDS, Londres, v.7, n.6, p.445-447, 1996.

LEHNINGER, A.L.; NELSON, D.L.; COX, M.M. Princípios de bioquímica. 2. ed. São Paulo: Sarvier, 1995. $839 \mathrm{p}$.

LESLIE, K. S.; GAFFNEY, K.; ROSS, C. N.; RIDLEY, S.; BARKER, T. H.; GARIOCH, J. J. A near fatal case of the dapsone hypersensitivity syndrome in a patient with urticarial vasculitis. Clin. Exp. Dermatol., Oxford, v.28, n.5, p.496, 2003.

MEISTER, A.; ANDERSON, M.E. Glutathione. Annu. Rev. Biochem., Palo Alto, v.52, p.711-760, 1993.

MEISTER, A. Glutathione deficiency produced by inhibition of its synthesis, and its reversal: Applications in research and therapy. Pharmacol. Ther., Oxford, v.51, p.155-194, 1991.

PAVITHRAN, K.; SATISH, T. C. Dapsone induced motor polyneuropathy in a patient with leprosy. Int. J. Lepr. Other Mycobact. Dis., Washington, v.65, n.2, p.262-263, 1997.
PRUSSICK, R.; ALI, M. A.; ROSENTHAL, D.; GUYATT, $\mathrm{G}$. The protective effect of vitamin $\mathrm{E}$ on the hemolysis associated with dapsone treatment in patients with dermatitis herpetiformis. Arch. Dermatol., Chicago, v.128, n.2, p.210-213, 1992.

ORGANIZAÇÃO PAN-AMERICANA DE SAÚDE (OPAS). Monitoramento da eliminação da hanseníase. Brasília, 2004. 27p.

QUEIROZ, R. H. C.; MELCHIOR, E. J.; SOUZA, A. M.; GOUVEIA, E.; BARBOSA, J. C.; CARVALHO, D. Haematological and biochemical alterations in leprosy patients already treated with dapsone and MDT. Pharm. Acta Helv., Zürich, v.72, p.209-213, 1997a.

QUEIROZ, R. H. C.; DREOSSI, S. A.; CARVALHO, D. A rapid, specific, and sensitive method for the determination of acetylation phenotype using dapsone. J. Anal. Toxicol., Niles, v.21, p.203-207, 1997b.

SALAMAT, A.; WATSON, H. G. Drug-induced methaemoglobinaemia presenting with angina follow the use of dapsone. Clin. Lab. Haematol., Oxford, v.25, n.5, p.327-328, 2003.

TINGLE, M. D.; COLEMAN, M. D.; PARK, B. K. An investigation of the role of metabolism in dapsone-induced methaemoglobinaemia using a two compartment in vitro test system. Br. J. Clin. Pharmacol., Londres, v.30, n.6, p.829-838, 1990.

WARD, K. E.; MCCARTY, M. W. Dapsone- induced methemoglobinemia. Ann. Pharmacother., Cincinnati, v.32, n.5, p.549-553, 1998.

Recebido para publicação em 10 de abril de 2007. Aceito para publicação em 21 de agosto de 2007. 